\title{
THE SYMMETRIC ALGEBRA OF QUOTIENTS OF AN ULTRAPRIME BANACH ALGEBRA
}

\author{
MARTIN MATHIEU
}

(Received 5 May 1989; revised 2 February 1990)

Communicated by $\mathbf{S}$. Yamamuro

\begin{abstract}
We present a symmetric version of a normed algebra of quotients for each ultraprime normed algebra. In addition, a $C^{*}$-algebra of quotients of an arbitrary $C^{*}$-algebra is introduced.

1980 Mathematics subject classification (Amer. Math. Soc.) (1985 Revision): primary 46 H 20; secondary, 46 L 05, 47 B 99, 16 A 08.
\end{abstract}

\section{Introduction}

A normed algebra $A$ over $\mathbb{C}$ is said to be ultraprime if

$$
\kappa:=\inf \left\{\left\|M_{a, b}\right\| \mid a, b \in A,\|a\|=\|b\|=1\right\}>0,
$$

where $M_{a, b}: x \mapsto a x b$ is a two-sided multiplication on $A$. In [9] we studied the basic properties of ultraprime normed algebras. In particular, every ultraprime algebra has trivial centre and its completion is an ultraprime Banach algebra (two of several features that distinguish ultraprime algebras from arbitrary prime normed algebras). It is also possible to enlarge every ultraprime algebra $A$ to a normable algebra of quotients, $Q(A)$; this one will again be ultraprime, and the restriction of the norm of $Q(A)$ to $A$ is equivalent to the original norm of $A$ [9, Theorem 4.1]. The construction of $Q(A)$ is in

(C) 1991 Australian Mathematical Society 0263-6115/91 \$A2.00+0.00

The greater part of this paper was completed while the author was a Visiting Fellow at the Centre for Mathematical Analysis of the Australian National University in Canberra. The support received from the CMA and the DFG is gratefully acknowledged. 
fact analogous to Martindale's ring of quotients for an arbitrary prime ring [6].

A symmetric version of the Martindale ring of quotients which works with balanced pairs of left and right module homomorphisms rather than with right module homomorphisms was introduced by Passman in [11] and computed in several cases (see also [10], in particular for the applications to the Galois theory of prime rings). Following his ideas, but using continuous module maps on ultraprime algebras, we will construct a normable symmetric algebra of quotients, $Q_{s}(A)$, in the sequel. Like $Q(A)$, this will be a normed algebra extension of the ultraprime normed algebra $A$, and the centre of $Q_{s}(A)$ coincides with the extended centroid of $A$. Also, $Q_{s}(A)$ is ultraprime.

Since $Q_{s}(A)$ carries a natural involution when $A$ is an involutive algebra, its completion will become a prime $C^{*}$-algebra if we start with a prime $C^{*}$ algebra $A$. Led by this, we define, for an arbitrary $C^{*}$-algebra $A$ and each filter $\mathscr{F}$ of closed essential ideals of $A$, a $C^{*}$-algebra of quotients $\widetilde{Q}_{s}(A ; \mathscr{F})$

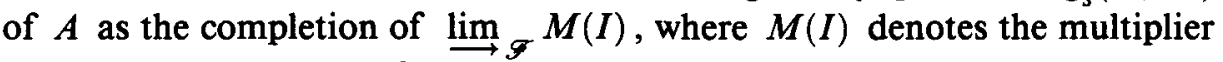
algebra of $I \in \mathscr{F}$. As $\widetilde{Q}_{s}(A ; \mathscr{F})=M(A)$ if $\mathscr{F}=\{A\}$, we may consider the $C^{*}$-algebra of quotients as a generalisation of the multiplier algebra of $A$. Some basic properties of $\widetilde{Q}_{s}(A ; \mathscr{F})$ will be discussed in Section 3.

While we were working on this paper we were sent a preprint by P. Ara [2] in which he also studies the extended centroid of a $C^{*}$-algebra $A$ and obtains a characterisation of it in terms of continuous functions on the primitive spectrum of $A$. We will compare his results with ours at the appropriate place below.

Note added. Shortly after submission of this paper, G. K. Pedersen informed the author that he used $M_{\text {loc }}(A)$ (see Definition 3.2 below), under the name 'essential multipliers', to study derivations of $C^{*}$-algebras in [Invent. Math. 45 (1978), 299-305]; see also [G. A. Elliott, J. Funct. Anal. 23 (1976), 1-10].

\section{Construction of the symmetric algebra of quotients}

Let $R$ be a ring. If $R$ is prime, then a unital prime ring of (right) quotients of $R$ was constructed by Martindale in [6]. If $R$ is semi-prime, this construction was extended by Amitsur in [1]. We will denote this ring of quotients by $Q_{a}(R)$. The centre $C$ of $Q_{a}(R)$ is the so-called extended centroid of $R$. Recently, the extended centroid of semi-prime normed algebras was studied by Cabrera Garcia and Rodriguez Palacios [4]. 
Throughout this section, $A$ will denote a non-zero ultraprime normed algebra over $\mathbb{C}$. It was proved in [9] that the extended centroid of $A$ is trivial and thus $A$ is a centrally closed algebra. Examples of ultraprime algebras are $\mathscr{K}(E)$ and $\mathscr{B}(E)$, the compact and the bounded linear operators on a normed space $E$, and every prime $C^{*}$-algebra [7]. G. Willis [15] proved that, if $G$ is either the free group on two generators or the group of all finite permutations on $\mathbb{N}$, then $l^{1}(G)$ is ultraprime. (On the other hand, if $G \neq\{1\}$ is a compact group or a locally compact abelian group, then $L^{1}(G)$ is never (ultra)prime.) Willis also raised the interesting question whether every amenable prime Banach algebra has to be ultraprime. An affirmative answer would have consequences on the automatic continuity of derivations, for example. We will denote the (unital ultraprime) normed algebra of quotients of $A$ introduced in [9] by $Q(A)$.

The purpose of this section is to present a symmetric version of $Q(A)$.

DEFINITION 2.1. Let $\mathscr{F}$ denote the lattice of all non-zero ideals of $A$, and let $\mathscr{F} \subseteq \mathscr{I}$ be any filter which is stable under products, that is, $I, J \in \mathscr{F}$ implies $I J \in \mathscr{F}$. For each $a \in A$ we denote by $L_{a}$ (respectively $R_{a}$ ) the left (respectively right) multiplication by $a$ (on $I \in \mathscr{F}$ ). Then $D C(\mathscr{F})$ is the set of all continuous double centralisers on $\mathscr{F}$, that is, an element in $D C(\mathscr{F})$ is a triple $\left(I, \rho_{l}, \rho_{r}\right)$ consisting of an ideal $I \in \mathscr{F}$ and continuous mappings $\rho_{l}, \rho_{r}$ from $I$ into $A$ satisfying $L_{x} \circ \rho_{l}=L_{\rho_{r}(x)}$ for every $x \in I$.

RemaRKs. 1. The 'associativity law' $L_{x} \circ \rho_{l}=L_{\rho_{r}(x)}$ can be replaced equivalently by $R_{x} \circ \rho_{r}=R_{\rho_{l}(x)}(x \in I)$.

2. We shall also allow that $\mathscr{F}=\{A\}$, even if $A \neq A^{2}$. In this case, $D C(\mathscr{F})$ is nothing but the set of all continuous double centralisers of $A$ as introduced by Johnson [5]. It follows from Definition 2.1, similarly as in [5] and for any filter $\mathscr{F}$, that both $\rho_{l}$ and $\rho_{r}$ are linear mappings, $\rho_{l}$ is a left centraliser, that is, $\rho_{l} \circ R_{a}=R_{a} \circ \rho_{l}$ for every $a \in A$, and $\rho_{r}$ is a right centraliser, that is, $\rho_{r} \circ L_{a}=L_{a} \circ \rho_{r}$ for every $a \in A$.

3. Each $a \in A$ yields a double centraliser via the 'double representation' [5, page 301] given by $a \mapsto\left(A, L_{a}, R_{a}\right)$.

We now define an equivalence relation $\sim$ on $D C(\mathscr{F})$ by

$$
\left(I, \rho_{l}, \rho_{r}\right) \sim\left(J, \sigma_{l}, \sigma_{r}\right) \text { if } \rho_{l \mid I \cap J}=\sigma_{l \mid I \cap J} \text { and } \rho_{r \mid I \cap J}=\sigma_{r \mid I \cap J} .
$$

The transitivity follows from the fact that, if $\rho_{l \mid U}=\sigma_{l \mid U}$ for some $U \in \mathscr{F}$ which is contained in their common domain $D$, then $\rho_{l \mid D}=\sigma_{l \mid D}$ (use the primeness of $A)$. In particular, $\left(I, \rho_{l}, \rho_{r}\right) \sim(I, 0,0)$ if and only if $\rho_{l}=\rho_{r}$ is not injective. Indeed, if $\rho_{l}(x)=0$ for some $0 \neq x \in I$ and $\rho_{l}=\rho_{r}$, then $\rho_{l}(A x A) \subseteq A \rho_{l}(x) A=0$ whence $\rho_{l}=0$. On $Q_{s}(A ; \mathscr{F}):=D C(\mathscr{F}) / \sim$ we 
define the following operations

$$
\begin{aligned}
{\left[\left(I, \rho_{l}, \rho_{r}\right)\right]+\left[\left(J, \sigma_{l}, \sigma_{r}\right)\right] } & :=\left[\left(I \cap J, \rho_{l}+\sigma_{l}, \rho_{r}+\sigma_{r}\right)\right], \\
{\left[\left(I, \rho_{l}, \rho_{r}\right)\right] \cdot\left[\left(J, \sigma_{l}, \sigma_{r}\right)\right] } & :=\left[\left(J I \cap I J, \rho_{l} \circ \sigma_{l}, \sigma_{r} \circ \rho_{r}\right)\right], \\
\lambda\left[\left(I, \rho_{l}, \rho_{r}\right)\right] & :=\left[\left(I, \lambda \rho_{l}, \lambda \rho_{r}\right)\right],
\end{aligned}
$$

where [.] denotes 'equivalence class' and $\lambda \in \mathbb{C}$.

Proposition 2.2. With the operations defined as above, $Q_{s}(A ; \mathscr{F})$ becomes a prime unital algebra, and the double representation induces a monomorphism $A \rightarrow Q_{s}(A ; \mathscr{F})$.

Proof. From the primeness of $A$, it is easy to verify that the operations are well-defined and satisfy the usual associativity and distributivity laws. The identity of $Q_{s}(A ; \mathscr{F})$ is the equivalence class of ( $A$, id, id), and the mapping $a \mapsto\left[\left(A, L_{a}, R_{a}\right)\right]$ is clearly an injective algebra homomorphism. We will henceforth consider $A$ as a subalgebra of $Q_{s}(A ; F)$. In order to prove that $Q_{s}(A ; \mathscr{F})$ is prime observe that for each $q \in Q_{s}(A ; \mathscr{F}) \backslash\{0\}$ there is $I \in \mathscr{F}$ such that $0 \neq q I \subseteq A$ and $0 \neq I q \subseteq A$. Indeed, take $\left(I, \rho_{l}, \rho_{r}\right) \in q$ and let $x \in I$. Then

$$
\begin{aligned}
q x & =\left[\left(I, \rho_{l}, \rho_{r}\right)\right] \cdot\left[\left(A, L_{x}, R_{x}\right)\right]=\left[\left(A I \cap I A, \rho_{l} \circ L_{x}, R_{x} \circ \rho_{r}\right)\right] \\
& =\left[\left(I^{2}, L_{\rho_{l}(x)}, R_{\rho_{l}(x)}\right)\right]=\rho_{l}(x) \in A,
\end{aligned}
$$

and similarly $x q=\rho_{r}(x) \in A$.

If $\rho_{l}(x)=0$ for all $x \in I$, then $\rho_{r}(y) a x=y \rho_{l}(a x)=0$ for all $y, x \in I$, $a \in A$ whence $\rho_{r}(y)=0$ for all $y \in I$. Thus, $q \neq 0$ implies both $\rho_{l} \neq 0$ and $\rho_{r} \neq 0$ which proves the claim.

Take now $q_{1}, q_{2} \in Q_{s}(A ; \mathscr{F}) \backslash\{0\}$ and $x_{1}, x_{2} \in A$ such that $q_{1} x_{1} \neq 0$ and $x_{2} q_{2} \neq 0$. Since $A$ is prime we find $z \in A$ with $q_{1} x_{1} z x_{2} q_{2} \neq 0$ which accomplishes the proof.

In order to endow $Q_{s}(A ; \mathscr{F})$ with an algebra norm we first note that the construction of $Q(A)$ in [9] can be extended easily from the case $\mathscr{F}=\mathscr{J}$ to general $\mathscr{F}$ and thus we will obtain an ultraprime normed algebra of quotients $Q(A ; \mathscr{F})$ with respect to $\mathscr{F}$. Our next aim is to realize $Q_{s}(A ; \mathscr{F})$ as a subalgebra of $Q(A ; \mathscr{F})$.

LeMMA 2.3. If $\left(I, \rho_{l}, \rho_{r}\right) \in q \in Q_{s}(A ; \mathscr{F})$, then $\kappa\left\|\rho_{r}\right\| \leq\left\|\rho_{l}\right\| \leq \kappa^{-1}\left\|\rho_{r}\right\|$. In particular, if $\kappa=1$, then $\left\|\rho_{l}\right\|=\left\|\rho_{r}\right\|$.

Proof. For all $x, y \in I$ we have $M_{\rho_{r}(x), y}=L_{\rho_{r}(x)} \circ R_{y}=L_{x} \circ \rho_{l} \circ R_{y}=$ $L_{x} \circ R_{y} \circ \rho_{l}$ and $M_{x, \rho_{l}(y)}=L_{x} \circ R_{\rho_{l}(y)} \stackrel{\rho_{r}}{=} L_{x} \circ R_{y} \circ \rho_{r}$. Therefore

$$
\kappa\left\|\rho_{r}(x)\right\|\|y\| \leq\left\|M_{\rho_{r}(x), y}\right\| \leq\|x\|\|y\|\left\|\rho_{l}\right\|,
$$


that is, $\kappa\left\|\rho_{r}\right\| \leq\left\|\rho_{l}\right\|$, and

$$
\kappa\|x\|\left\|\rho_{l}(y)\right\| \leq\left\|M_{x, \rho_{l}(y)}\right\| \leq\|x\|\|y\|\left\|\rho_{r}\right\|,
$$

that is, $\kappa\left\|\rho_{l}\right\| \leq\left\|\rho_{r}\right\|$.

The purely algebraic version of $Q_{s}(A ; \mathscr{F})$, which we will denote by $Q_{a s}(A ; F)$, is built up from equivalence classes of not necessarily continuous double centralisers defined on ideals in $\mathscr{F}$. This is Passman's symmetric ring of quotients. (In fact, the construction in [11] for $\mathscr{F}=\mathscr{I}$ differs slightly from ours; however it yields the same result.) The following consequence of Lemma 2.3 shows how $Q_{s}(A ; \mathscr{F})$ 'sits inside' $Q_{a s}(A ; \mathscr{F})$.

CoRollary 2.4. If $q \in Q_{a s}(A ; \mathscr{F})$ contains a representative $\left(I, \rho_{l}, \rho_{r}\right)$ with either $\rho_{l}$ or $\rho_{r}$ continuous, then $q \in Q_{s}(A ; \mathscr{F})$.

Proof. If $\rho_{l}$ is bounded, then so is $\rho_{r}$ and vice versa, as follows from (1) and (2) in the proof of Lemma 2.3. If $\left(J, \sigma_{l}, \sigma_{r}\right)$ is another representative of $q$, then $\sigma_{l}$ and $\sigma_{r}$ are continuous by [9, Section 4, Remark 3].

The next result is the analogue of [11, Proposition 1.6].

Proposition 2.5. The mapping $\left[\left(I, \rho_{l}, \rho_{r}\right)\right] \mapsto\left[\left(I, \rho_{l}\right)\right]$ is an isomorphism from $Q_{s}(A ; \mathscr{F})$ into $Q(A, \mathscr{F})$, and considering $Q_{s}(A ; \mathscr{F})$ thus as a subalgebra of $Q(A ; \mathscr{F})$ we have

$$
Q_{s}(A ; \mathscr{F})=\{q \in Q(A ; \mathscr{F}) \mid \exists I \in \mathscr{F}: I q \subseteq A\} .
$$

Proof. It is clear from the definition that $\left[\left(I, \rho_{l}, \rho_{r}\right)\right] \mapsto\left[\left(I, \rho_{l}\right)\right]$ is a well-defined algebra homomorphism into $Q(A ; \mathscr{F})$, which is injective since $\rho_{l}=0$ implies $\rho_{r}=0$ (see above). Identifying $Q_{s}(A ; \mathscr{F})$ with its image in $Q(A ; \mathscr{F})$, we already noticed that, for $q \in Q_{s}(A ; \mathscr{F})$, there is $I \in \mathscr{F}$ such that $I q \subseteq A$. Conversely, suppose $q \in Q(A ; \mathscr{F})$ and $I \in \mathscr{F}$ satisfy $I q \subseteq A$ and, by replacing $I$ by a smaller ideal in $\mathscr{F}$, if necessary, $q I \subseteq A$. Then, by putting $\rho_{r}(x)=x q \quad(x \in I)$ we obtain a right centraliser on $I$, which is continuous by 2.4 , such that

$$
\rho_{r}(x) y=(x q) y=x(q y)=x \rho_{l}(y) \text { for all } x, y \in I .
$$

Thus, $\left[\left(I, \rho_{l}, \rho_{r}\right)\right]=q \in Q_{s}(A ; \mathscr{F})$.

DEFINITION 2.6. Equipped with the norm of $Q(A ; \mathscr{F})$ (that is, $\|q\|=$ $\left.\inf \left\{\left\|\rho_{l}\right\| \mid\left(I, \rho_{l}, \rho_{\mathrm{r}}\right) \in q\right\}\right), Q_{s}(A ; \mathscr{F})$ is called the symmetric normed algebra of quotients of $A$ with respect to $\mathscr{F}$. If $\mathscr{F}=\mathscr{F}$, we write $Q_{s}(A)$ instead of $Q_{s}(A ; \mathscr{I})$ and speak of the symmetric normed algebra of quotients. 
THEOREM 2.7. Let $A$ be an ultraprime normed algebra. Then $Q_{s}(A ; \mathscr{F})$ is an ultraprime normed subalgebra of $Q(A ; \mathscr{F})$, and the restriction of the norm of $Q_{s}(A ; F)$ to $A$ is equivalent to the original norm of $A$. Moreover, the centre of $Q_{s}(A)$ coincides with the extended centroid of $A$.

Proof. By Propositions 2.2 and 2.5 and [9, Theorem 4.1], $Q_{s}(A ; \mathscr{F})$ is both a normed subalgebra of $Q(A ; \mathscr{F})$ and a normed algebra extension of $A$, since $\|a\| \geq\left\|\left[\left(I, L_{a}, R_{a}\right)\right]\right\| \geq \kappa\|a\|$ for each $a \in A$. In the remainder of this proof, we will denote the original norm of $a \in A$ by $\|a\|_{A}$. Take $p, q \in$ $Q_{s}(A ; \mathscr{F})$ with $\|p\|=\|q\|=1$. By definition, there are $\left(I, \rho_{l}, \rho_{r}\right) \in p$ and $\left(J, \sigma_{l}, \sigma_{r}\right) \in q$ such that $\left\|\rho_{l}\right\| \geq 1$ and $\left\|\sigma_{l}\right\| \geq 1$. By Lemma 2.3, $\left\|\sigma_{r}\right\| \geq \kappa$. Hence, for each $0<\varepsilon<1$, there are $x \in I,\|x\|_{A}=1, y \in J,\|y\|_{A}=\kappa^{-1}$ such that $\|p x\|_{A}=\left\|\rho_{l}(x)\right\|_{A} \geq 1-\varepsilon$ and $\|y q\|_{A}=\left\|\sigma_{r}(y)\right\|_{A} \geq 1-\varepsilon$. For every $z \in A$ with $\|z\|_{A}=1$ we have

$$
\kappa\|p x z y q\|_{A} \leq\|p x z y q\| \leq\left\|M_{p, q}\right\|\|x z y\| \leq \kappa^{-1}\left\|M_{p, q}\right\|,
$$

and thus

$$
\begin{aligned}
\kappa^{2}(1-\varepsilon)^{2} & \leq \kappa^{2}\|p x\|_{A}\|y q\|_{A} \leq \kappa \sup \left\{\|p x z y q\|_{A} \mid z \in A,\|z\|_{A}=1\right\} \\
& \leq \kappa^{-1}\left\|M_{p, q}\right\| .
\end{aligned}
$$

This shows that $\left\|M_{p, q}\right\| \geq \kappa^{3}>0$, that is, $Q_{s}(A ; \mathscr{F})$ is ultraprime.

In order to prove the last assertion let $q \in Z\left(Q_{s}(A)\right)$, the centre of $Q_{s}(A)$. For every $x \in I$ with $\left(I, \rho_{l}, \rho_{r}\right) \in q$ we have $\rho_{l}(x)=q x=x q=\rho_{r}(x)$. Thus, $\rho_{l}=\rho_{r}$ is an $A$-bimodule homomorphism corresponding uniquely to $\left[\left(I, \rho_{l}\right)\right]$ in the extended centroid $C$. Conversely, every element in $C$ has only $A$-bimodule homomorphisms as representatives and these are continuous by [9, Lemma 4.3]. It follows that $C=Z\left(Q_{s}(A)\right)$.

It will not be surprising that $Q_{s}(A)$ as well as $Q(A)$ have an abstract characterisation by some kind of 'maximality'.

Proposition 2.8. The normed algebra of quotients $Q(A)$ is the maximal normed algebra extension $Q$ of $A$ satisfying the following conditions:

(i) for each $q \in Q$ there is $I \in \mathcal{F}$ such that $q I \subseteq A$;

(ii) if $q \in Q, I \in \mathscr{I}$ satisfy $q I=0$, then $q=0$.

The symmetric normed algebra of quotients $Q_{s}(A)$ is the maximal normed algebra extension $Q_{s}$ of $A$ satisfying conditions (i), (ii) and

(i') for each $q \in Q_{s}$ there is $I \in \mathscr{I}$ such that $I q \subseteq A$.

Proof. It is clear that $Q(A)$ (respectively $Q_{s}(A)$ ) satisfy (i), (ii) (respectively (i), (ii) and (i')) (compare with the proof of 2.2 ). Suppose that 
$\left(Q,\|\cdot\|^{\prime}\right)$ is a normed algebra containing $A$ as a subalgebra such that $\gamma_{1}\|a\| \leq\|a\|^{\prime} \leq \gamma_{2}\|a\|$ holds for all $a \in A$ and some $\gamma_{1}, \gamma_{2}>0$. If, for every $q \in Q$, there is $I \in \mathscr{F}$ with $q I \subseteq A$, we define $\rho_{l}: I \rightarrow A$ by $\rho_{l}(x)=q x$ $(x \in I)$. Clearly, $\rho_{l}$ is a left centraliser, and $\rho_{l}$ is continuous since

$$
\left\|\rho_{l}(x)\right\|=\|q x\| \leq \gamma_{1}^{-1}\|q x\|^{\prime} \leq \gamma_{1}^{-1}\|q\|^{\prime}\|x\|^{\prime} \leq \gamma_{1}^{-1} \gamma_{2}\|q\|^{\prime}\|x\|
$$

for all $x \in I$. Now define an algebra homomorphism $Q \rightarrow Q(A)$ by $q \mapsto\left[\left(I, \rho_{l}\right)\right]$ which is injective in the presence of (ii) and continuous since $\left\|\left[\left(I, \rho_{l}\right)\right]\right\| \leq\left\|\rho_{l}\right\| \leq \gamma_{1}^{-1} \gamma_{2}\|q\|^{\prime}$. In the case $Q_{s}$, we proceed analogously and obtain a continuous left (respectively right) centraliser $\rho_{l}$ (respectively $\rho_{r}$ ) by $\rho_{l}(x)=q x, \rho_{r}(x)=x q \quad(x \in I)$ if $q \in Q_{s}$ and $I \in \mathscr{I}$ satisfy, without restriction of generality, $q I \cup I q \subseteq A$. As

$$
L_{\rho_{r}(x)}=L_{x q}=L_{x} \circ L_{q}=L_{x} \circ \rho_{l} \text { for all } x \in I,
$$

we have $\left(I, \rho_{l}, \rho_{r}\right) \in D C(\mathscr{S})$ and we obtain a continuous embedding $Q_{s} \rightarrow$ $Q_{s}(A), q \mapsto\left[\left(I, \rho_{l}, \rho_{r}\right)\right]$.

We conclude this section with some additional comments on the continuity of double centralisers. To this end, let $\left(\rho_{l}, \rho_{r}\right)$ be a double centraliser from a non-zero ideal $I$ into $A$. Similar to [5, Theorem 14] one shows that both $\rho_{l}$ and $\rho_{r}$ are closable mappings, and by exploiting the ultraprimeness that their closures define another double centraliser $\left(\tilde{\rho}_{l}, \tilde{\rho}_{r}\right)$ on $\tilde{I} \in \mathscr{I}$ contained in $\bar{I}$, the closure of $I$ in $A$. Therefore, $q \in Q_{a s}(A ; \mathscr{F})$ always contains closed representatives, and if $A$ is complete, then $\tilde{I}=\bar{I}$ if and only if $\rho_{l}, \rho_{r}$ are continuous. In an ultraprime Banach algebra, we can thus equally well start with closed ideals and arbitrary double centralisers and, after taking equivalence classes, obtain $Q_{s}(A ; \mathscr{F})$.

REMARK. The left handed version of $Q(A ; \mathscr{F})$ is constructed by using continuous right centralisers in an analogous way, and thus gives no additional information in general. In concrete cases, however, one side may be distinguished from the other. For instance, if $A=\mathscr{K}(E)$, the compact operators on a Banach space $E$, and $\mathscr{F}=\{A\}$, then $Q_{a}(A)=Q(A)=Q_{a s}(A)=$ $Q_{s}(A)=\mathscr{B}(E)$ by [5, Corollary on page 313], whereas the (continuous) right centraliser algebra is isometrically anti-isomorphic with $\mathscr{B}\left(E^{*}\right)$ and coincides with $Q_{s}(A)$ if and only if $E$ is reflexive [5, page 314].

\section{The $C^{*}$-algebra of quotients}

The completion of $Q_{s}(A ; \mathscr{F})$ is again ultraprime by [9, Proposition 3.5]. For every ultraprime Banach algebra $A$, we therefore have obtained the symmetric ultraprime Banach algebra of quotients $\widetilde{Q}_{s}(A ; \mathscr{F})$ of $A$ (with 
respect to $\mathscr{F}$ ). In this section, we turn our attention to the particular case of $C^{*}$-algebras.

Let $A$ be a prime $C^{*}$-algebra. Then, by [7, Proposition 2.3], $A$ is ultraprime with $\kappa=1$. Let $\mathscr{F}$ be any filter of closed ideals in $\mathscr{F}$; then, $D C(\mathscr{F})$ is invariant under the mapping $\left(I, \rho_{l}, \rho_{r}\right) \mapsto\left(I, \rho_{r}^{*}, \rho_{l}^{*}\right)$, where $\rho_{r}^{*}(x):=\rho_{r}\left(x^{*}\right)^{*}, \rho_{l}^{*}(x):=\rho_{l}\left(x^{*}\right)^{*}$ for $x \in I$. By means of this, $Q_{s}(A ; \mathscr{F})$ becomes an involutive algebra satisfying

$$
\begin{aligned}
x^{*} q^{*} & =\left[\left(I, L_{x^{*}} \circ \rho_{r}^{*}, \rho_{l}^{*} \circ R_{x^{*}}\right)\right]=\left[\left(I, L_{\rho_{l}^{*}\left(x^{*}\right)}, R_{\rho_{l}^{*}\left(x^{*}\right)}\right)\right] \\
& =\rho_{l}^{*}\left(x^{*}\right)=\rho_{l}(x)^{*}=(q x)^{*},
\end{aligned}
$$

if $\left(I, \rho_{l}, \rho_{r}\right) \in q$ and $x \in I$, and thus

$$
\left\|q^{*} q\right\| \geq\left\|x^{*} q^{*} q x\right\|=\left\|(q x)^{*} q x\right\|=\|q x\|^{2},
$$

if $x \in I,\|x\| \leq 1$.

Hence, $\left\|q^{*} q\right\| \geq\|q\|^{2}$ and therefore the norm of $Q_{s}(A ; \mathscr{F})$ satisfies the $C^{*}$-condition. By the remarks at the beginning of this section, $\widetilde{Q}_{s}(A ; \mathscr{F})$ is thus a unital prime $C^{*}$-algebra. Since $\kappa=1, \widetilde{Q}_{s}(A ; \mathscr{F})$ contains $A$ isometrically and, by Lemma 2.3 and $\left[9\right.$, Lemma 4.5], $\|q\|=\left\|\rho_{l}\right\|=\left\|\rho_{r}\right\|$ for every representative $\left(I, \rho_{l}, \rho_{r}\right)$ of $q \in Q_{s}(A ; \mathscr{F})$.

We may view $\widetilde{Q}_{s}(A ; \mathscr{F})$ in the following alternative way. Each element $\left(I, \rho_{l}, \rho_{r}\right)$ in $D C(\mathscr{F})$ corresponds uniquely to a multiplier on $I$, since $I=I^{2}$ (cf. $\left.[12,3.12]\right)$. Conversely, each element $z$ in $M(I)$, the multiplier algebra of $I$, gives rise to a continuous double centralizer $\left(I, L_{z}, R_{z}\right)$. Considering $\mathscr{F}$ directed downwards by inclusion, we define isometric embeddings $M(I) \rightarrow M(J)$, if $J \subseteq I$, by restricting the centralisers to $J$.

It is now evident that the following result holds.

Proposition 3.1. Let $A$ be a prime $C^{*}$-algebra and let $\mathscr{F}$ be a filter of non-zero closed ideals of $A$. Then, $Q_{s}(A ; \mathscr{F})$ is isometrically "-isomorphic to $\lim _{\mathscr{F}} M(I)$.

This observation leads us to introduce the following concept.

Definition 3.2. Let $A$ be a $C^{*}$-algebra, let $\mathscr{I}_{c e}$ denote the lattice of closed essential ideals in $A$ and $\mathscr{F} \subseteq \mathscr{I}_{c e}$ be any filter. If $I, J \in \mathscr{I}_{c e}$ and $J \subseteq I$, then $M(I)$ can be considered as a $C^{*}$-subalgebra of $M(J)$. Using these embeddings we define $Q_{s}(A ; \mathscr{F}):=\underline{\lim }_{\mathscr{F}} M(I)$, and the completion $\widetilde{Q}_{s}(A ; \mathscr{F})$ of this direct limit is called the $C^{*}$-algebra of quotients of $A$ (with respect to $\mathscr{F})$. If $\mathscr{F}=\{A\}$, then $Q_{s}(A ; \mathscr{F})=M(A)$, the multiplier algebra of $A$. We therefore call $\widetilde{Q}_{s}\left(A ; \mathscr{J}_{c e}\right)$ the local multiplier algebra of $A$ and denote it henceforth by $M_{\mathrm{loc}}(A)$. 
Our terminology is further justified by the following characterisation of $M_{\mathrm{loc}}(A)$.

Proposition 3.3. The local multiplier algebra $M_{\mathrm{loc}}(A)$ of a $\mathbb{C}^{*}$-algebra $A$ is the largest $C^{*}$-algebra $B$ which contains $A$ as a $C^{*}$-subalgebra and for each $I \in \mathscr{I}_{c e}$ a $C^{*}$-subalgebra $B_{I}$ in which $I$ is an essential ideal such that $\cup B_{I}$ is dense in $B$.

We omit the easy proof (compare with Proposition 2.8). The situation may be pictured by the following sequence of natural inclusions.

$$
I \rightarrow A \rightarrow M(A) \rightarrow M(I) \rightarrow I^{* *} \rightarrow A^{* *}
$$

for every $I \in \mathscr{I}_{c e}$, where $A^{* *}$ denotes the enveloping $W^{*}$-algebra of $A$. This is immediate from [12, 3.12.8 and 3.7.9].

A $C^{*}$-algebra $A$ is prime if and only if $M(A)$ is prime. Taking $M_{\mathrm{loc}}(A)$, we can slightly improve on this statement.

Propostion 3.4. The following conditions on a $C^{*}$-algebra $A$ are equivalent.

(a) $A$ is prime.

(b) $M_{\mathrm{loc}}(A)$ is prime.

(c) $Z\left(M_{\text {loc }}(A)\right)$ is one-dimensional.

Proof. If $A$ is prime, then $Q_{s}\left(A ; \mathscr{I}_{c e}\right)=Q_{s}(A)$ is ultraprime by Theorem 2.7, and hence $M_{\mathrm{loc}}(A)$ is prime by Proposition 3.1 and [9, Proposition 3.5]. This proves $(\mathrm{a}) \Rightarrow(\mathrm{b})$, and (b) $\Rightarrow$ (c) is clear. To prove (c) $\Rightarrow$ (a) suppose that $A$ is not prime. Take non-zero closed ideals $I_{0}, J_{0}$ in $A$ that are maximal with respect to the property $I_{0} \cap J_{0}=I_{0} J_{0}=0$. Then $I_{0}+J_{0}$ is a closed essential ideal. Indeed, if $\left(I_{0}+J_{0}\right) \cap J=0$ for some closed ideal $J$ of $A$, then $I_{0} \cap\left(J_{0}+J\right)=0$ so that by maximality $J_{0}=J_{0}+J$. But $J_{0} \cap J=0$ whence $J=0$. We now define $x, y \in M\left(I_{0}+J_{0}\right)$ by $L_{x}=R_{x}=$ projection onto $I_{0}$ and $L_{y}=R_{y}=$ projection onto $J_{0}$. Clearly, $x, y \in Z\left(M\left(I_{0}+J_{0}\right)\right)$, the centre of the multiplier algebra, and $x y=0$. If $I \subseteq I_{0}+J_{0}$, then $Z\left(M\left(I_{0}+J_{0}\right)\right) \subseteq Z(M(I))$ whence $x, y \in Z(\stackrel{\lim }{\longrightarrow} M(I))=\underline{\lim } Z(M(I))$ (we identify $x, y$ with their images in the direct limit). From this we infer that $x, y$ are non-zero divisors of zero in $Z\left(M_{\mathrm{loc}}(A)\right)$, which is a commutative $C^{*}$-algebra and therefore has dimension greater than one.

REMARKs AND ExAMPLES. 1. It follows from the above proof that, for $A$ being prime, it suffices that $C_{b}:=Z\left(Q_{s}\left(A ; \mathscr{J}_{c e}\right)\right)=\underset{\longrightarrow}{\longrightarrow} Z(M(I))$ is prime. We shall call $C_{b}$ the bounded extended centroid of $A$. If $A$ is not prime, then 
$C_{b}$ is in general strictly smaller than $C$, the extended centroid of $A$. For an example, let $A=l^{\infty}(\mathbb{N})$ and $I=\varphi_{0}$, the non-closed essential ideal of finite sequences. By $f\left(\left(\xi_{n}\right)_{n \in \mathbb{N}}\right)=\left(n \xi_{n}\right)_{n \in \mathbb{N}}$ we can then define a discontinuous $A$-bimodule homomorphism $f: I \rightarrow A$; hence, $[(I, f, f)] \notin C_{b}$.

2. In [2], Ara uses the canonical correspondence between closed (essential) ideals of a $C^{*}$-algebra $A$ and open (dense) subsets of the primitive spectrum $\check{A}$ of $A$ as well as the Dauns-Hofmann theorem to obtain a description of $C_{b}$ (respectively $C$ ) as follows. For every topological space $X$ let $C_{b}(X)$ (respectively $C(X)$ ) denote the space of all bounded continuous (respectively all continuous) complex-valued functions on $X$. If $U, V \in \mathscr{D}$, the open dense subsets of $\check{A}, C(U)$ can be embedded into $C(V)$, if $V \subseteq U$. Then, $C_{b} \cong \lim _{\mathscr{D}} C_{b}(U)$ while $C \cong \lim _{\mathscr{D}} C(U)$ [2, Proposition 2.2 and Theorem 2.3]. Ara's approach is rather more of a global nature than ours; for example, he obtains our starting point, viz. that the extended centroid of a prime $C^{*}$ algebra is trivial [7, Proposition 2.5], as a consequence of his main theorem [2, Corollary 2.4]. Another main difference is his use of the fact that the norm in a $C^{*}$-algebra is determined by the order to identify $Q_{s}\left(A ; \mathscr{I}_{c e}\right)$ as the 'bounded part' of $Q_{a s}\left(A ; \mathscr{I}_{c e}\right)$ [2, Theorem 1.3], and he also does not consider the completion $\widetilde{Q}_{s}\left(A ; \mathscr{I}_{c e}\right)$.

3. If $A$ is a simple $C^{*}$-algebra, then clearly $M(A)=M_{\text {loc }}(A)$. More generally, the same holds if $\breve{A}$ is discrete. If $A$ is a factor and $I$ is a non-zero closed ideal, similar arguments to those in [9, Examples in Section 4] show that $M_{\text {loc }}(I)=M_{\text {loc }}(A)=A$. It is clear that a $C^{*}$-algebra $A$ is commutative if and only if $M_{\mathrm{loc}}(A)$ is commutative. In this case, $M_{\mathrm{loc}}(A)=$ $C^{*}-\lim _{\longrightarrow} C(\beta \hat{I})=C\left(\lim _{\longleftarrow} \hat{I}\right)$ where $\hat{I}$ is the spectrum of $I \in \mathscr{J}_{c e}$ (cf. [14, 11.8.7]). Observe, however, that $\lim _{\longleftarrow} \beta \hat{I} \neq \beta \underset{\lim }{\leftarrow}$, since the Stone-Čech functor $\beta$ is not inversely continuous.

4. It is natural to ask for conditions implying that $Q_{s}(A ; \mathscr{F})$ is already complete. For example, if $A$ is a prime $C^{*}$-algebra which is not antiliminal, then it contains a minimal closed ideal [8, Propositions 2.2 and 2.3]; thus, the intersection of any sequence of non-zero closed ideals is non-zero. It follows by [13, Theorem 2] that $Q_{s}(A)=M_{\text {loc }}(A)$. However, this is not typical for type I $C^{*}$-algebras as the following example shows. Let $A=C(\mathbb{I})$, where $\mathbb{I} \subseteq \mathbb{R}$ is the unit interval. For each $n \in \mathbb{N}$ let $I_{n}$ denote the closed essential ideal consisting of those functions which vanish on the set of the $2^{n-1}$ dyadic numbers $\frac{1}{2^{n}}, \ldots, \frac{2^{n}-1}{2^{n}}$ together with 0 and 1 . Then, $\bigcap_{n \in \mathbb{N}} \hat{I}_{n}$ is dense, but not open, and $\bigcap_{n \in \mathrm{N}} I_{n}=0$.

The last example is extended in the next proposition which shows in particular that the commutative counterexample arises from the lack of isolated points in the spectrum. 
Proposition 3.5. Let $A$ be a $C^{*}$-algebra with non-zero socle. Then $Q_{s}\left(A ; \mathscr{I}_{c e}\right)$ is complete.

Proof. If the socle of $A$, that is, the sum of all minimal left ideals, is non-zero, then there exists a minimal projection $p$ in $A$ [3, BA.4]. Let $H_{p}=$ $(A p,(\cdot \cdot))$ where the inner product is defined by $(x \mid y) p=y^{*} x, x, y \in A p$. Then the left regular representation induces an irreducible representation $\pi_{p}$ of $A$ on $H_{p}$, and $t=\pi_{p}^{-1}(0)$ is the unique primitive ideal not containing $p\left[3, C^{*} .4 .2\right]$. Therefore, $\{t\}=\check{A} \backslash \operatorname{hull}(\{p\})$ is open in $\check{A}$, that is, $t$ is an isolated point. Hence, $\{t\} \subseteq \check{I}$ whenever $I \in \mathscr{I}_{c e}$ and thus $t \subseteq I$.

Suppose that there is $J \in \mathscr{I}_{c e}$ such that $J \subseteq t$; then, $J=t$ and $t$ is essential. If there is no such $J$, every $I \in \mathscr{I}_{c e}$ contains $\overline{A p A}$ : if $s \in \check{A}$, $s \neq t$, then $p \in s$. Hence, each closed ideal $K \nsubseteq t$ contains $p$ since $K=\operatorname{ker}(\operatorname{hull}(K))[12,3.13 .8]$ and therefore the closed ideal generated by $p$. The ideal $t+\overline{A p A}$ is essential since $K \nsubseteq t$ implies $K \cap(t+\overline{A p A}) \supseteq \overline{A p A} \neq 0$ and $K \subseteq t$ implies $K \cap(t+\overline{A p A}) \supseteq K$. We conclude that in either case there is an ideal $t^{\text {ess }} \in \mathscr{I}_{c e}$ which contains $t$ and is contained in each other $I \in \mathscr{I}_{c e}$.

Let $\left(q_{n}\right)_{n \in \mathbb{N}}$ be a Cauchy sequence in $Q_{s}\left(A ; \mathscr{I}_{c e}\right)$ and $I_{n} \in \mathscr{I}_{c e}$ be such that $q_{n} \in M\left(I_{n}\right)$. Then $q_{n} \in M\left(t^{\text {ess }}\right)$ for all $n$ and, since $M\left(t^{\text {ess }}\right)$ is complete, $q_{n} \rightarrow q \in M\left(t^{\text {ess }}\right) \subseteq Q_{s}\left(A ; \mathscr{F}_{c e}\right)$. (This is in fact the argument in [13, Theorem 2] adapted to our situation.)

We note that the existence of minimal projections is not necessary for the completeness of $Q_{s}\left(A ; \mathscr{I}_{c e}\right)$ as the example of the Calkin algebra $\mathscr{E}(H)$ shows. (This remark also applies to the non-separable case since each closed ideal in $\mathscr{C}(H)$ is the image of a closed ideal in $\mathscr{B}(H)$ and the closed ideals in $\mathscr{B}(H)$ are well-ordered.)

The structure of $M_{\mathrm{loc}}(A)$ seems to be rather interesting and deserves further investigation. We conclude this paper with another extension of a well known result for $M(A)$; it also generalises (a) $\Leftrightarrow$ (b) in Proposition 3.4.

Proposition 3.6. For every pair of elements $a, b \in M_{\mathrm{loc}}(A)$ the norm of $M_{a, b}$ on $M_{\mathrm{loc}}(A)$ can be computed as

$$
\left\|M_{a, b}\right\|=\sup \{\|a x b\| \mid x \in A,\|x\|=1\} .
$$

Proof. Suppose that $a, b \in Q_{s}\left(A ; \mathscr{I}_{c e}\right)$ and let $\varepsilon>0$. Since $Q_{s}\left(A ; \mathscr{I}_{c e}\right)$ is dense in $M_{\mathrm{loc}}(A),\left\|M_{a, b}\right\|=\left\|M_{a, b \mid Q_{s}\left(A ; \mathcal{F}_{c e}\right)}\right\|$. Thus, there is $x \in M(K)$ for some $K \in \mathscr{I}_{c e}$ such that $\|x\|=1$ and $\|a x b\| \geq(1-\varepsilon)\left\|M_{a, b}\right\|$. Take $I, J \in \mathscr{I}_{c e}$ such that $a \in M(I), b \in M(J)$; then $a, b, x \in M(I \cap J \cap K)$, 
so, in order to simplify the notation, we may assume $I=J=K$. Since $I \subseteq M(I) \subseteq I^{* *}$ and $\left\|M_{a, b \mid I}\right\|=\left\|\left(M_{a, b \mid I}\right)^{* *}\right\|$, we deduce that

$$
\left\|M_{a, b \mid I}\right\|=\left\|M_{a, b \mid M(I)}\right\| \geq\|a x b\| \geq(1-\varepsilon)\left\|M_{a, b}\right\| .
$$

This shows that $\sup \{\|a x b\| \mid x \in A,\|x\|=1\} \geq\left\|M_{a, b}\right\|$.

Suppose now that $a, b \in \widetilde{Q}_{s}\left(A ; \mathscr{I}_{c e}\right)$ and let $\varepsilon>0$. There are $a^{\prime}, b^{\prime} \in$ $Q_{s}\left(A ; \mathscr{I}_{c e}\right)$ and $x \in A,\|x\|=1$ such that $\left\|M_{a, b}-M_{a^{\prime}, b^{\prime}}\right\|<\varepsilon$ and $\left\|a^{\prime} x b^{\prime}\right\| \geq\left\|M_{a^{\prime}, b^{\prime}}\right\|-\varepsilon$. Thus,

$$
\left\|M_{a, b}\right\| \leq\left\|M_{a^{\prime}, b^{\prime}}\right\|+\varepsilon \leq\left\|a^{\prime} x b^{\prime}\right\|+2 \varepsilon \leq\|a x b\|+3 \varepsilon
$$

which proves $\left\|M_{a, b}\right\| \leq \sup \{\|a x b\| \mid x \in A,\|x\|=1\}$. The converse inequality is clear since $A \subseteq M_{\mathrm{loc}}(A)$.

\section{References}

[1] S. A. Amitsur, 'On rings of quotients', Sympos. Math. 8 (1972), 149-164.

[2] P. Ara, 'The extended centroid of $C^{*}$-algebras', Arch. Math., 54 (1990), 358-364.

[3] B. A. Barnes, G. J. Murphy, M. R. F. Smyth and T. T. West, Riesz and Fredholm Theory in Banach algebras, (Pitman Research Notes in Math. 67, Boston, Mass., 1982).

[4] M. Cabrera Garcia and A. Rodriguez Palacios, 'Extended centroid and central closure of semiprime normed algebras. A first approach', Comm. Algebra, to appear.

[5] B. E. Johnson, 'An introduction to the theory of centralizers', Proc. London Math. Soc. 14 (1964), 299-320.

[6] W. S. Martindale, 'Prime rings satisfying a generalized polynomial identity', J. Algebra 12 (1969), 576-584.

[7] M. Mathieu, 'Elementary operators on prime $C^{*}$-algebras, I', Math. Ann. 284 (1989), 223-244.

[8] M. Mathieu, 'Elementary operators on prime $C^{*}$-algebras, II', Glasgow Math. J. 30 (1988), 275-284.

[9] M. Mathieu, Rings of quotients of ultraprime Banach algebras. With applications to elementary operators, (Proc. Centre Math. Anal. Austral. Nat. Univ. 21, 1989, 297317).

[10] D. S. Passman, Group rings, crossed products and Galois theory, (CBMS Series no. 64, Amer. Math. Soc., Providence, R.I., 1986).

[11] D. S. Passman, 'Computing the symmetric rings of quotients', J. Algebra 105 (1987), 207-235.

[12] G. K. Pedersen, $C^{*}$-algebras and their automorphism groups, (Academic Press, London 1979).

[13] J. S. Pym, 'Inductive and projective limits of normed spaces', Glasgow Math. J. 9 (1968), 103-105. 
[14] Z. Semadeni, Banach spaces of continuous functions, (Polish Scientific Publishers, Warsaw, 1971).

[15] G. Willis, private communication. See also: Ultraprime group algebras, (Proc. Centre Math. Anal. Austral. Nat. Univ. 21, 1989, pp. 345-349).

\section{Mathematisches Institut \\ Universität Tübingen \\ Auf der Morgenstelle 10 \\ D-7400 Tübingen \\ Federal Republic of Germany}

